\title{
Acute arrest of hematopoiesis induced by infection with Staphylococcus epidermidis following total knee arthroplasty: A case report and literature review
}

\author{
LINTAO BI* ${ }^{*}$ JUN LI* ${ }^{*}$ ZHENXIA LU, HUI SHAO and YING WANG \\ Department of Hematology and Oncology, China-Japan Union Hospital Affiliated to Jilin University, \\ Changchun, Jilin 130033, P.R. China
}

Received November 10, 2014; Accepted November 25, 2015

DOI: $10.3892 /$ etm.2016.3023

\begin{abstract}
Infection is one of the most severe complications of total knee prosthesis implantation. The present study reported the case of a 74-year-old female that developed a Staphylococcus epidermidis infection following a cemented total knee arthroplasty. A routine blood test revealed neutropenia and anemia, while $S$. epidermidis was detected in the peripheral blood and bone marrow. In the present case, $S$. epidermidis infection led to acute arrest of hematopoiesis (AAH), also known as aplastic crisis, which is the temporary cessation of red cell production. The development of AAH secondary to $S$. epidermidis infection is rare and, to the best of our knowledge, this is the first case reported in the literature. The present study increased our knowledge of this rare disease and its characteristics, which will enable physicians to be aware of the development of AAH as a rare complication of S. epidermidis infection.
\end{abstract}

\section{Introduction}

Total knee arthroplasty (TKA) is one of the most common orthopedic procedures and is an effective treatment for patients with severe osteoarthritis of the knees that allows pain relief and improved function and quality of life (1). However, infection is a common complication of the implementation of total knee prosthetics, and the current incidence of prosthetic knee infection is $1-3 \%$ (2). The most frequent microorganism involved is Staphylococcus aureus (3).

Correspondence to: Professor Zhenxia Lu, Department of Hematology and Oncology, China-Japan Union Hospital Affiliated to Jilin University, 126 Xiantai Street, Changchun, Jilin 130033, P.R. China

E-mail: luzx2013@126.com

${ }^{*}$ Contributed equally

Key words: Staphylococcus epidermidis, total knee arthroplasty, infection
Staphylococcus epidermidis was commonly described as relatively innocuous, but has been recognized as an important opportunistic pathogen, being the most frequent cause of device-associated infections in hospital (4). Overall, 20-36\% infections in TKA are caused by methicillin-resistant staphylococci, with methicillin-resistant Staphylococcus aureus and methicillin-resistant $S$. epidermidis being increased $(5,6)$ It has been reported that the treatment success rate is only $\sim 18 \%$ when the associated bacteria is multi-drug resistant $(6,7)$. The present case study described a case of $S$. epidermidis infection, which developed following cemented TKA. S. epidermidis infection led to acute arrest of hematopoiesis (AAH) in the present case. AAH, also known as aplastic crisis, is the temporary cessation of red cell production. A previous study reported that infection may trigger the process of $\mathrm{AAH}$, and exposure to certain drugs may be the possible etiology (8). The patient presented with a high fever, pallor and tiredness. Routine blood test revealed neutropenia and anemia and $S$. epidermidis was detected in the peripheral blood and bone marrow. The aim of the present study was to describe a case of TKA that resulted in infection with $S$. epidermidis infection and subsequent development of AHH. Its objective is to enable physicians to be aware of AAH, a rare complication of patients infected with S. epidermidis. To the best of our knowledge, this is the first such case reported in the literature.

\section{Case report}

A 74-year-old Chinese female patient was referred to the Department of Orthopedics at the China-Japan Union Hospital Affiliated to Jilin University (Changchun, China) on June the $13^{\text {th }}, 2014$. The patient presented with fever, restricted movement, pain and swelling of the knee, which developed 4 months after left TKA surgery due to gonarthrosis. The patient was receiving regular medical treatment for hypertension for 5 years (oral administration $30 \mathrm{mg}$ nifedipine once/day; Bayer AG, Leverkusen, Germany) and had previously undergone a right TKA due to gonarthrosis 4 years prior to the present admission. Preoperative X-ray scans of the patient's left knee are shown in Fig. 1A. Following admission, the left knee was aspirated and the fluid was sent for culture. 

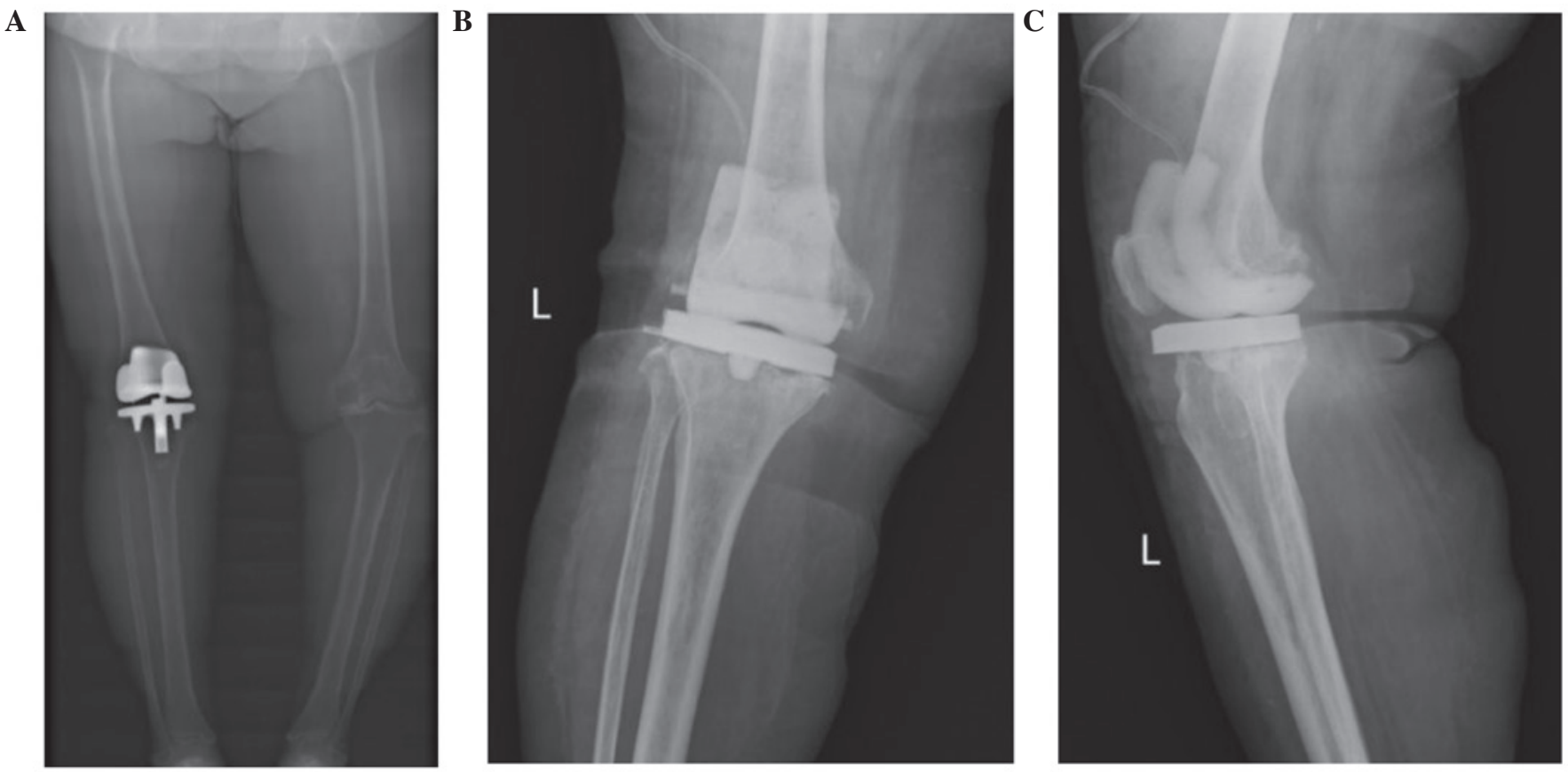

Figure 1. (A) Pre-operative X-rays of the patient's left knee. (B and C) X-ray scans of the patient's left knee following installation of a drainage tube into the articular cavity.
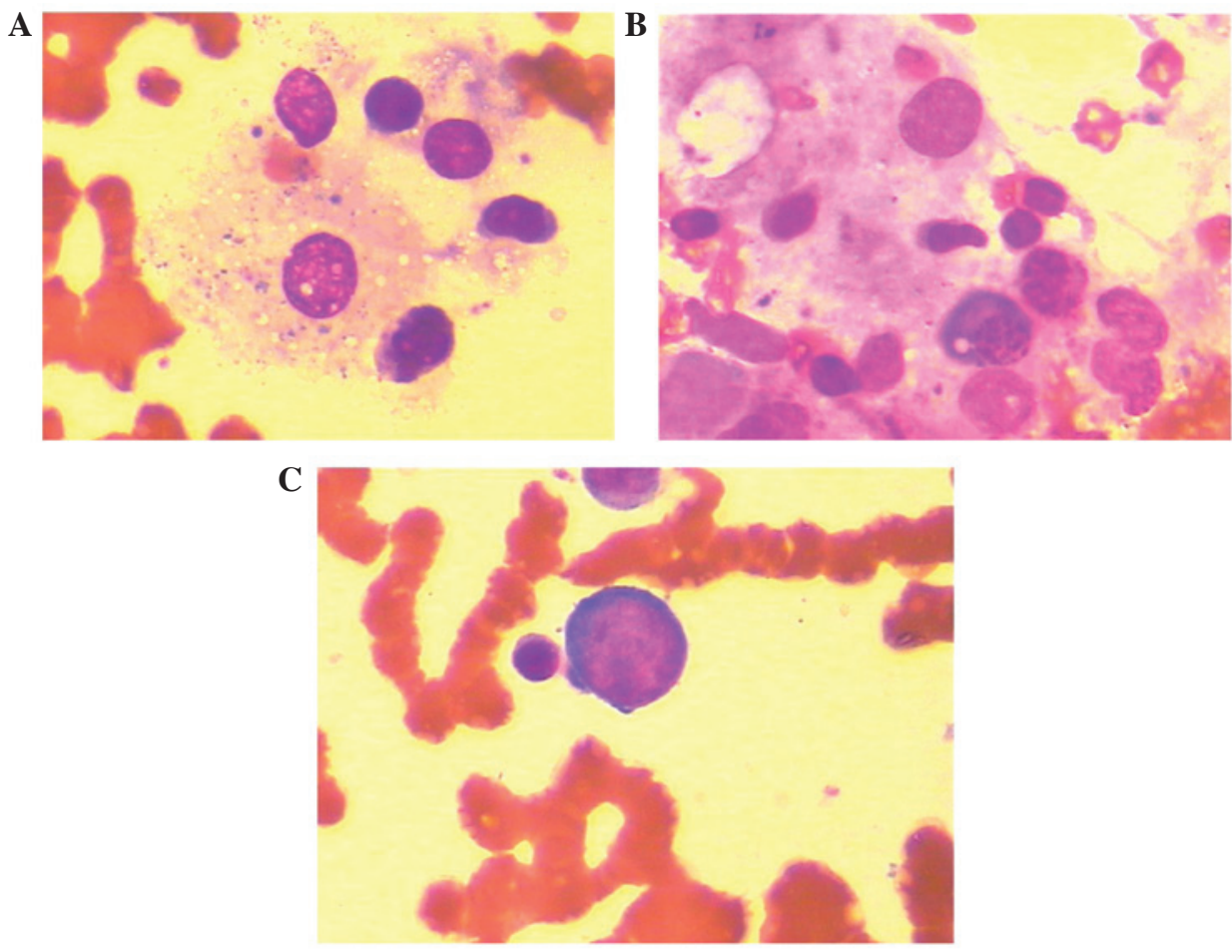

Figure 2. Bone marrow aspiration demonstrating the predominance of non-hematopoietic cells, including (A) lymphocytes, histocytes and (B) plasma cells, and $(\mathrm{C})$ a giant pronormoblast. Magnification, $\mathrm{x} 1,000$.

Prior to the culture results, the patient was administered vancomycin (intravenous administration $1 \mathrm{~g} / 12 \mathrm{~h}$ for 18 days; Elli Lilly and Company, Indianapolis, IN, USA) via intravenous and intra-articular injection. Several germicultures of the joint fluid were negative. Routine blood tests and analysis of the renal and liver function were normal upon admission. However, additional testing revealed an elevated C-reactive protein level (129 mg/l; normal range, 0-8 mg/l) and erythrocyte sedimentation rate $(64 \mathrm{~mm} / \mathrm{h})$. X-ray scans were then performed following implantation of a drainage tube into the articular cavity (Fig. 1B and C). As no improvement was observed following antibiotic therapy and drainage, the components of the prosthetic knee were removed. The temperature of the patient was reduced to $<38^{\circ} \mathrm{C}$ during the first postoperative 
week; however, the patient presented with an increased fever (body temperature, $39.8^{\circ} \mathrm{C}$ ) once again in the second postoperative week. At 3 weeks postoperatively, routine blood tests demonstrated a reduced leucocyte count and anemia: White blood cell (WBC) count, $0.5 \times 10 \% / 1$ (granulocytes, $7 \%$; lymphocytes, $88 \%$; and monocytes, $5 \%$; normal range, $4-10 \times 10^{9} / 1$ ); red blood cell count, $2.52 \times 10^{12} / 1$ (normal range, $3.5-5 \times 10^{12} / 1$ ); hemoglobin concentration, $7.8 \mathrm{~g} / \mathrm{dl}$ (normal range, $97 \mathrm{~g} / \mathrm{l}$ ); hematocrit, $22.4 \%$ (normal range, $0.35-0.47 \%$ ); mean corpuscular volume, $88.6 \mathrm{fl}$ (normal range, 80-100 fl); and platelet count, $143 \times 10^{9} / 1$ (normal range, $100-300 \times 10^{9} / 1$ ). The reticulocyte count had decreased to $0 \%$ (normal range, $0.5-1.5 \%$ ). A subsequent bone marrow smear demonstrated marked diminution of hematopoietic cells (Fig. 2). Megakaryocytes were not detected. Predominantly, non-hematopoietic cells, were detected, including stromal cells, plasma cells, macrophages and infiltrate of lymphoid cells, while giant pronormoblasts were clearly visible. The results of the peripheral blood and bone marrow cultures were compatible with the diagnosis of AAH (9). The patient was subsequently transferred to the Department of Hematology and Oncology at the China-Japan Union Hospital Affiliated to Jilin University on July the $25^{\text {th }}$, 2015. Blood and bone marrow cultures detected the growth of S. epidermidis (methicillin-resistant strain), which was subsequently demonstrated to be susceptible to vancomycin and tigecycline (Pfizer Inc., New York, NY, USA). According to the results of the antibiotic sensitivity test, the patient was treated with vancomycin and a supportive treatment, which consisted of nutritional support (intravenous administration of $10 \mathrm{~g}$ albumin/day for 5 days; Octapharma AG, Lachen, Switzerland), defervescence (oral administration of $500 \mathrm{mg}$ acetaminophen when fever is $>38.5^{\circ} \mathrm{C}$; Beijing Shuguang Pharmaceutical Industrial Co., Ltd., Beijing, China), and bone marrow hematopoiesis stimulation (hypodermic injection of $300 \mu \mathrm{g}$ G-CSF once/day for 12 days; Changchun Jinsai Pharmaceutical Co. Ltd., Jilin, China). However, the symptoms persisted after 1 week of treatment and the WBC count had reduced to $0.1 \times 10^{9}$ cells $/ 1$. Furthermore, the patient exhibited left-sided heart failure and brain natriuretic peptide (BNP) levels increased to $35,000 \mathrm{pg} / \mathrm{ml}$; therefore, the patient was administered cardiotonic (three intravenous bolus injections of $200 \mathrm{mg}$ cedilanid; Xudong Haipu Pharmaceutical Co., Ltd, Shanghai, China) and diuretics (eight intravenous bolus injections of $20 \mathrm{mg}$ lasix; Nantong Jinghua Pharmaceutical Co., Ltd., Nantong, China). The patient also presented with a cough, without expectoration. The results of the $(1 \rightarrow 3)-\beta$-D-glucanemia test $(10)$ indicated a level of $(1 \rightarrow 3)-\beta$-D-glucanemia was $1,509.1 \mathrm{pg} / \mathrm{ml}$, which was markedly elevated (normal range, 100.5-151.5 pg/ml). Therefore, the patient was diagnosed with a deep mycotic infection and administered vancomycin (intravenous administration of $1 \mathrm{~g} / 12 \mathrm{~h}$ for 17 days; Eli Lilly and Company), meropenem (intravenous administration of $0.5 \mathrm{~g} / 8 \mathrm{~h}$ for 8 days; Sumitomo Dainippon Pharma Co., Ltd., Tokyo, Japan) and voriconazole (02 g/12 h for 10 days; Pfizer, Inc.) triple anti-inflammatory treatment. After 1 week, the patient's temperature and reticulocyte count returned to normal, while the WBC count increased to $15 \times 10^{9}$ cells/l; therefore, meropenem and voriconazole treatment was stopped. However, the left-sided heart failure persisted following treatment and the BNP levels remained elevated. Renal failure was subsequently detected, since the serum creatinine level was $229 \mathrm{umol} / 1$ and blood urea nitrogen (BUN) was $21.5 \mathrm{mmol} / \mathrm{l}$. Reexamination of the blood cultures demonstrated the growth of $S$. epidermidis (methicillin-resistant); therefore, antibiotic treatment was amended from vancomycin to tigecycline (intravenous administration of $100 \mathrm{mg} /$ day on day 1 then $50 \mathrm{mg} / 12 \mathrm{~h}$ on days 2 and 3; Pfizer, Inc.). However, renal failure persisted and several days later the patient presented with anuria as the renal function continued to deteriorate (serum creatinine, $520 \mathrm{umol} / \mathrm{l}$; BUN, $40.8 \mathrm{mmol} / \mathrm{l}$; and serum potassium, $5.7 \mathrm{mmol} / \mathrm{l})$. The patient succumbed to infection with $S$. epidermidis and the secondary renal and heart failure 25 days after admission to the Department of Hematology and Oncology.

\section{Discussion}

AAH is defined as a transient episode of pure red cell aplasia characterized by the absence of reticulocytes in the peripheral blood, the absence of erythroid precursors and elevated giant pronormoblasts in the bone marrow (11-13). It has previously been reported that infection or drug exposure may trigger AAH (8). AAH predominantly occurs in patients with hemolytic anemia and, as it is a self-limiting disease, aplasia usually persists for 5-10 days $(12,13)$. Routine blood tests typically demonstrate anemia and a decreased reticulocyte count, and although pancytopenia does not usually occur, mild leukopenia and/or thrombocytopenia are occasionally detected (11). In the present patient, the complete blood count demonstrated neutropenia and moderate anemia. Although the hematopoietic activity was recovered, the patient eventually succumbed to infection with $S$. epidermidis and the secondary renal and heart failure.

The most common cause of severe aplastic crisis for patients with chronic hemolytic anemia is infection with parvovirus B19 $(11,14)$. However, rare cases of severe aplastic anemia due to acute parvovirus B19 infection have been reported in previously healthy individuals $(15,16)$. In addition to parvovirus B19, there are various viral infections that contribute to AAH, including the Epstein Barr virus, cytomegalovirus, HIV and rubella $(17,18)$. Additional triggering agents in transient aplastic crises include pharmacological agents (such as methotrexate) and irradiation $(18,19)$. Patients typically present with increased fatigue, pallor, activity intolerance or shortness of breath. For the vast majority of patients with acute aplastic anemia, only supportive and symptomatic treatment can be offered. Transfusion of red blood cells ( $\mathrm{RBC}$ ) is required to relieve the symptoms of anemia for certain patients.

The present study described a case of AAH caused by S. epidermidis infection which appeared following the application of cemented TKA. Periprosthetic joint infection (PJI) is one of the most severe complications following TKA. This is a difficult issue for orthopaedic surgeons, as well as for patients and their families, due to its challenging management and the impact on the patients' quality of life $(3,20)$.

Epidemiologic studies investigating various trends in the microbiological profile of PJI between two referral centers in Europe and the United States demonstrated that PJI caused by methicillin-resistant $S$. aureus is becoming 
increasingly prevalent $(21,22)$. In the present case, growth of methicillin-resistant $S$. epidermidis was detected in blood and bone marrow cultures; however, a germiculture of joint fluid remained negative following various repeats. Biofilm formation may explain the phenomenon of culture-negative PJI. Novel methods, including DL-dithiothreitol and polymerase chain reaction using various probes, are being developed in order to improve the ability of microbiological culture in detecting the pathogenic causes of PJI $(23,24)$.

Wu et al (25) conducted a case-control study in order to investigate the effects of patient factors on the risk of PJI in a Chinese population. The results demonstrated that an increased risk of PJI in this Chinese population was associated with: Diabetes; age of 65-75 years; body-mass index of $\geq 28 \mathrm{~kg} / \mathrm{m}^{2}$; a history of alcohol abuse; and residence in rural areas. For the present patient, old age and obesity may have been influential risk factors.

The present patient was not receiving any medication that could trigger transient aplastic crises, and had no history of irradiation therapy. Therefore, it is hypothesized that the cause of AAH was infection with S. epidermidis. The pathogenesis of acute aplastic anemia induced by $S$. epidermidis infection is yet to be elucidated.

In conclusion, the present study described a case of AAH induced by S. epidermidis infection, which developed following cemented TKA. AAH induced by methicillin-resistant $S$. epidermidis infection is a rare and life-threatening complication. The present study increased our knowledge of this rare disease and its characteristics, which will enable physicians to be aware of the development of AAH as a rare complication of S. epidermidis infection. Further studies are required in order to elucidate the exact pathogenesis of AAH.

\section{References}

1. Vahedian-Ardakani M, Mortazavi S and Farzan M: Total knee arthroplasty: Does the tibial medial side defect affect outcome? Acta Med Iran 8: 462-465, 2015.

2. Soriano A, Bori G, García-Ramiro S, Martinez-Pastor JC, Miana T, Codina C, Maculé F, Basora M, Martínez JA Riba J, et al: Timing of antibiotic prophylaxis for primary total knee arthroplasty performed during ischemia. Clin Infect Dis 46: 1009-1014, 2008.

3. Martínez-Pastor JC, Maculé-Beneyto F and Suso-Vergara S: Acute infection in total knee arthroplasty: Diagnosis and treatment. Open Orthop J 7: 197-204, 2013.

4. Otto M. Molecular basis of Staphylococcus epidermidis infections. Semin Immunopathol 34: 201-214, 2012.

5. Peersman G, Laskin R, Davis J and Peterson M: Infection in total knee replacement: A retrospective review of 6489 total knee replacements. Clin Orthop Relat Res 392: 15-23, 2001.

6. Joshy S, Gogi N, Thomas B,Mahale A and Singh BK: Delayed onset of deep infection after total knee arthroplasty: Comparison based on the infecting organism. J Orthop Surg (Hong Kong) 15: 154-158, 2007.
7. Kilgus DJ, Howe DJ and Strang A: Results of periprosthetic hip and knee infections caused by resistant bacteria. Clin Orthop Relat Res 404: 116-124, 2002.

8. Lee JH, Lee JH, Shin YR, Lee JS, Kim WK, Chi HS, Park CJ and Lee KH: Spontaneous remission of aplastic anemia: A retrospective analysis. Haematologica 86: 928-933, 2001.

9. Yan ZS, Zhang L, Wang HJ, Zhou K, Li JP, Huang ZD, Wan L, Shang L, Bao XL and Zhang FK: Acute arrest of hemopoiesis mimics aplastic anemia: 23 cases report. Zhonghua Xue Ye Xue Za Zhi 28: 750-753, 2007 (In Chinese).

10. Marty FM and Koo S: Med Mycol. Role of (1-->3)-beta-Dglucan in the diagnosis of invasive aspergillosis. Med Mycol 47 Suppl 1: S233-S240, 2009.

11. Borsato ML, Bruniera P, Cusato MP, Spewien KE, Durigon EL and Toporovski J: Aplastic crisis in sickle cell anemia induced by parvovírus B19. J Pediatr (Rio J) 76: 458-460, 2000 (In Portuguese).

12. Ilesanmi OO: Pathological basis of symptoms and crises in sickle cell disorder: Implications for counseling and psychotherapy. Hematol Rep 26: e2, 2010.

13. Cauff BE and Quinn CT: Transient parvovirus-associated hypoplasia of multiple peripheral blood cell lines in children with chronichemolytic anemia. Pediatr Blood Cancer 50: 861-864, 2008.

14. Carzavec D, Gaćina P, Vasilj A and Katović SK: Aplastic crisis induced by human parvovirus B19 as an initial presentation of hereditary spherocytosis. Coll Antropol 34: 619-621, 2010.

15. Al-Abdwani RM, Khamis FA, Balkhair A, Sacharia M and Wali YA: A child with human parvovirus B19 infection induced aplastic anemia and acute hepatitis: Effectiveness of immunosuppressive therapy. Pediatr Hematol Oncol 25: 699-703, 2008.

16. Kaptan K, Beyan C, Ural AU, Ustün C, Cetin T, Avcu F, Kubar A, Aliş M and Yalçin A: Successful treatment of severe aplastic anemia associated with human parvovirus B19 and Epstein-Barr virus in a healthy subject with allo-BMT. Am J Hematol 67: 252-255, 2001.

17. Leoz Gordillo I and Pérez Suárez E: Aplastic crisis due to Parvovirus B19 and Epstein-Barr virus in a patient with hereditary spherocytosis. An Pediatr (Barc) 82: e102-e107, 2015.

18. Rajput R, Sehgal A, Jain D, Sen R and Gupta A: Acute parvovirus B19 infection leading to severe aplastic anemia in a previously healthy adult female. Indian J Hematol Blood Transfus 28: 123-126, 2012

19. Zhou Yan and Zhu Shuqing: Acute arrest of hematopoiesis due to oral methotrexate in a patient with uremia. Adverse Drug Reactions J 12: 351-352, 2010.

20. Alijanipour P and Parvizi J: Infection post-total knee replacement: Current concepts. Curr Rev Musculoskelet Med 7: 96-102, 2014.

21. Bjerke-Kroll BT, Christ AB, McLawhorn AS, Sculco PK, Jules-Elysée KM and Sculco TP: Periprosthetic joint infections treated with two-stage revision over 14 years: An evolving microbiology profile. J Arthroplasty 29: 877-882, 2014.

22. Aggarwal VK, Bakhshi H, Ecker NU, Parvizi J, Gehrke T and Kendoff D: Organism profile in periprosthetic joint infection: Pathogens differ at two arthroplasty infection referral centers in Europe and in the United States. J Knee Surg 27: 399-406, 2014.

23. Drago L, Signori V, De Vecchi E, Vassena C, Palazzi E, Cappelletti L, Romanò D and Romanò CL: Use of dithiothreitol to improve the diagnosis of prosthetic joint infections. J Orthop Res 31: 1694-1699, 2013.

24. Rak M, Barlič-Maganja D, Kavčič M, Trebše R and Cőr A: Comparison of molecular and culture method in diagnosis of prosthetic joint infection. FEMS Microbiol Lett 343: 42-48, 2013.

25. Wu C, Qu X, Liu F, Li H, Mao Y and Zhu Z: Risk factors for periprosthetic joint infection after total hip ar oplasty and total knee arthroplasty in Chinese patients. PLoS One 9: e95300, 2014. 